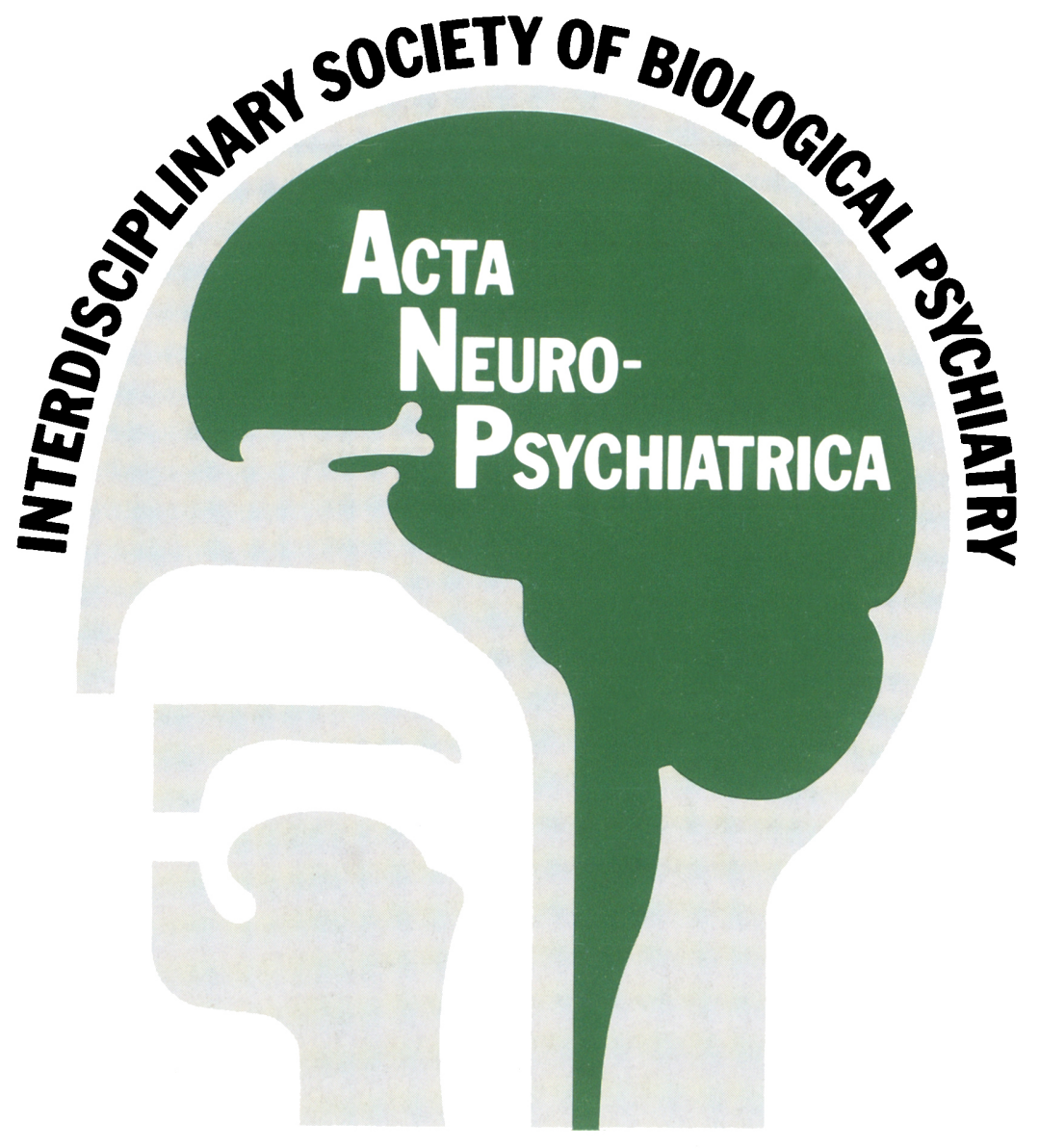

Acta NeUROPSYChIATRICA

OFFICIEEL WETENSCHAPPELIJK ORGAAN VAN HET I G B P (INTERDISCIPLINAIR GENOOTSCHAP VOOR BIOLOGISCHE PSYCHIATRIE)

2 JAARGANG NUMMER 3 - SEPTEMBER 1990

Psychiatrische symptomatologie en leeftijdsafhankelijkheid J.A.C. Oosterwijk, E.J.Colon

Neuropsychiatrische betekenis van corticosubcorticale relaties W. van den Bergh

De rol van serotonine bij gewoonte-geweld en impulsiviteit: een overzicht M.D. Virkkunen

De tyramineconjugatie test en depressie

J.G. Storosum, F. de Jonghe, H.F.A. Schalken, J. Huyser, H.N. Sno

Psychofarmakologika IV

J.Korf 


\section{resocialiserende neuroleptica}

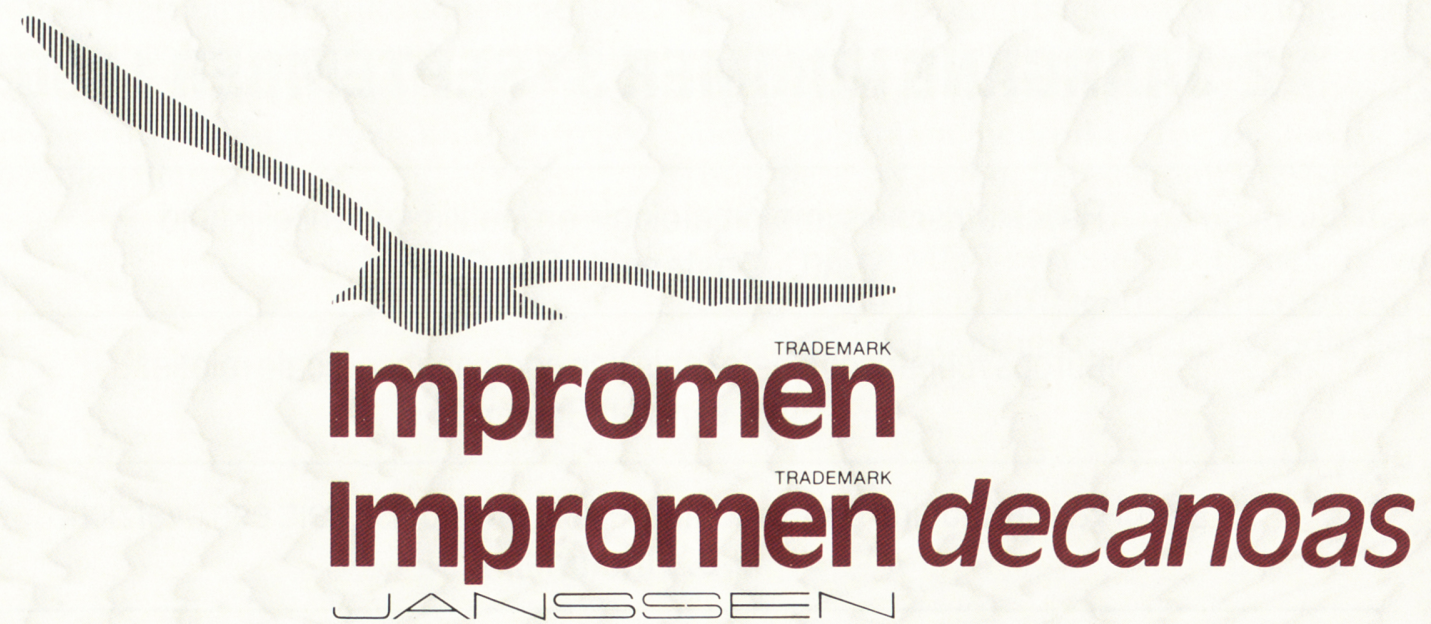




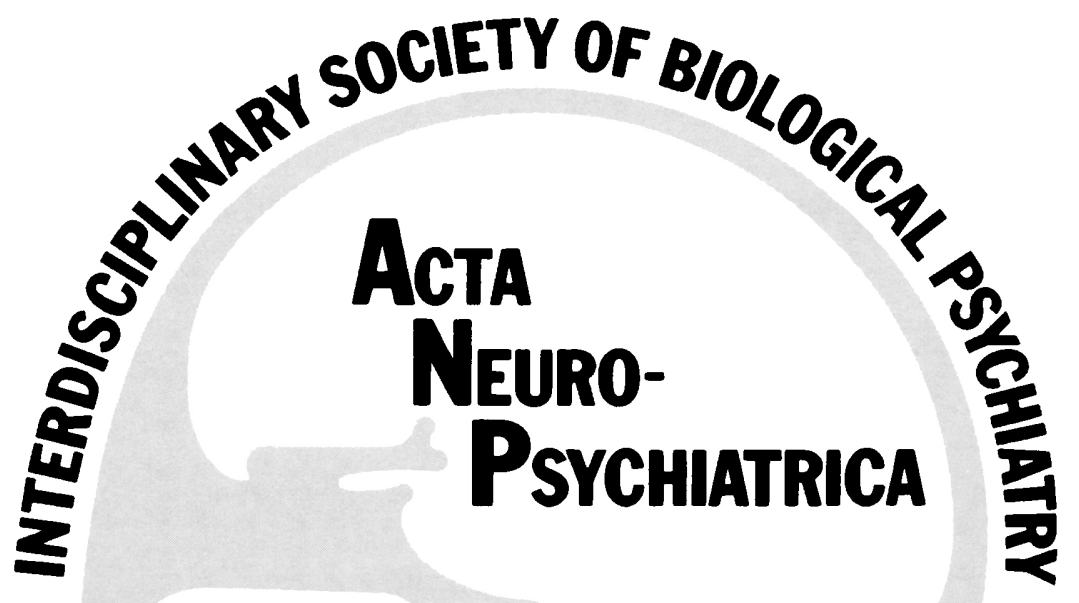

ACta Neuropsychiatrica

OFFICIEEL WETENSCHAPPELIJK ORGAAN VAN HET I G B P

(INTERDISCIPLINAIR GENOOTSCHAP VOOR BIOLOGISCHE PSYCHIATRIE)

VOLUME 2, NUMBER 3 - SEPTEMBER 1990

\section{CONTENTS}

From the editor

From the board

Psychiatric symptoms and age dependency: a preliminary A.M.D.P.-IV study in 140 psychogeriatic patients. J.A.C. Oosterwijk, E.J. Colon

Neuropsychiatry of corticosubcortical relations: aphasia and depression as paradigmata.

W. van den Bergh

Serotoninergic findings in habitual violence and impulsivity: a review.

M.D. Virkkunen

The tyramine conjugation test and depression

J.G. Storosum, F. de Jonghe, H.F.A. Schalken, J. Huyser, H.N.Sno

Psychopharmacologics IV

J.Korf 


\section{ACTA NEUROPSYCHIATRICA}

Officieel orgaan van het Interdisciplinair Genootschap voor Biologische Psychiatrie

ISSN 0924-2708

Reed Healthcare Communications bv,

Reaal 2, 2353 TL Leiderdorp,

Postadres: Postbus 182,

2350 AD Leiderdorp.

Tel.: 071-415151.

Fax: 31-(0)71-414923.

\section{Redactie}

Prof. dr. A.R. Cools

Dr. H.J.A. de Cuyper

Dr. H.A.H. D'haenen

Prof. dr. J. Korf

Prof. dr. F.H.M. Verhey

\section{Eindredactie}

Dr. M.J.A.J.M. Hoes

Redactiesecretaris

A.P. Van de Voort

Redactiesecretariaat

Postbus 199, 9200 AD Drachten.

Tel.: 31-(0)5128-1485.

Fax:31-(0)5128-1818.

Advertenties: Voor alle inlichtingen;

Reed Healthcare Communications bv,

Reaal 2, 2353 TL Leiderdorp.

Tel.: 071-415151.

Postadres: Postbus 182,

2350 AD Leiderdorp.

Abonnementen: Kunnen ieder moment worden aangegaan voor minimaal één jaar en worden stilzwijgend telkens voor een jaar verlengd, indien niet minimaal zes weken voor afloop een opzegging is ontvangen.

Voor Nederland $f 60$,- incl. BTW.

Voor België Bfrs. 1500,-p.a.; psychiaters in opleiding Bfrs. 1000,-.

Leden van het IGBP en psychiaters in opleiding ontvangen Acta Neuropsychiatrica gratis.

Overige landen US \$30,- p.a. + verzendkosten.

Abonnementsgeld overmaken naar giro 4368301, ten name van Reed Healthcare Communications bv, Reaal 2, 2353 TL Leiderdorp.
Losse nummers: $f$ 20,- per stuk excl. BTW en verzendkosten.

Frequentie $4 \mathrm{x}$ per jaar.

Copyright: Reed Healthcare Communications bv. Niets uit deze uitgave mag worden gefotocopieerd en/of verveelvoudigd zonder voorafgaande toestemming van de uitgever.

Acta Neuropsychiatrica is het officiële orgaan van het Interdisciplinair Genootschap voor Biologische Psychiatrie (IGBP).

Redactie-adviesraad

Prof. dr. H. Van Den Berghe, Leuven

Prof. dr. B. Bohus, Haren

Prof. dr. R.J. van den Bosch, Groningen

Prof. dr. C.H.M. Brunia, Tilburg

Prof. dr. E.J. Colon, Poortugaal

Prof. dr. P. Cosyns, Edegem

Dr. M. Danhof, Leiden

Dr. M.P. Derde, Brussel

Prof. dr. J. Godderis, Leuven

Dr. W.P. Haaijman, Lent

Prof. dr. R.H. van den Hoofdakker, Groningen

Dr. C. Hooijer, Amsterdam

Prof. dr. B. van Houdenhove, Leuven

Prof. dr. J. Jolles, Maastricht

Prof. dr. L. Kaufman, Brussel

Drs. G.A.S. Koster van Groos, Rosmalen

Prof. dr. M. Kuilman, Utrecht

Dr. A.J.M. Loonen, Vught

Em. prof.dr. A. Löwenthal, Antwerpen

Dr. P. Moleman, Rotterdam

Prof. dr. M. Niermeyer, Rotterdam

Prof. dr. R. Nieuwenhuys, Nijmegen

Prof. dr. S.J. Nijdam, Milsbeek

Prof. dr. J. Peuskens, Kortenberg

Prof. dr. J.M. van Ree, Utrecht

Prof. dr. J. De Roeck, Edegem

Dr. C.J. Slooff, Groningen

Prof. dr. E. Thierry, Gent

Prof. dr. F. Tilders, Amsterdam

Prof. dr. D.H.G. Versteeg, Utrecht

Dr. T.B. Vree, Nijmegen

Dr. T.A.C.M. van Woerkom, Den Haag 